\title{
ON TWO THEOREMS OF GIBBS
}

BY PAUL SAUREL

Among the many results contained in Gibbs's famous memoir are two important equations. ${ }^{x}$ The first of these is a relation between the changes of pressure and temperature in a univariant system; the second is a relation between the changes of pressure, temperature, and chemical potential in a divariant system, and this equation, when certain conditions are satisfied, takes a form analogous to that of the first. Gibbs shows, in the special case of one component, that his equation for univariant systems is equivalent to the Clapeyron-Clausius formula.

The object of the present note is to show that, in the general case of $n$ components, Gibbs's equation for univariant systems is equivalent to the Clapeyron-Clausius formula, and that the equation for bivariant systems can, when the conditions referred to above are satisfied, be likewise put into the form of the Clapeyron-Clausius equation.

For this purpose we shall start with the following equation, which is established by Gibbs :

$$
v^{\prime} d p=\eta^{\prime} d t+m_{1}^{\prime} d \mu_{1}+m_{2}^{\prime} d \mu_{2}+\cdots+m_{n}^{\prime} d \mu_{n} .
$$

In this equation $p$ denotes the pressure, $t$ the temperature, $v^{\prime}, \eta^{\prime}$ denote the volume and the entropy of the first phase, $\mu_{x}, \mu_{2}, \ldots \mu_{n}$ the chemical potentials of the $n$ independent components of the system, and $m^{\prime}, m^{\prime}{ }_{2} \cdots m_{n}^{\prime}$ the masses of these components contained in the first phase. For each of the $r$ phases there is an equation similar to ( $\mathrm{r}$ ).

Let us denote by $\mathrm{M}_{i}$ the mass of the $i$-th phase, by $v_{i}, \eta_{i}$ the volume and the entropy of the unit of mass of the $i$-th phase, by I 32.

' On the Equilibrium of Heterogeneous Substances. Equations I29 and 
$m_{i j}$ the mass of the $j$-th component which is contained in the unit of mass of the $i$-th phase, and by $9 \pi_{j}$ the total mass of the $j$-th component. Moreover, let us denote the volume and the entropy of the system by $\mathrm{V}$ and $\mathrm{H}$.

If we divide the equation which corresporids to the $i$-th phase by $\mathrm{M}_{i}$, and if we make use of the foregoing notation, we shall obtain :

$$
\begin{array}{r}
v_{i} d p=\eta_{i} d t+m_{i \mathrm{I}} d \mu_{\mathrm{t}}+m_{i 2} d \mu_{i}+\cdots+m_{i n} d \mu_{n}, \\
{[i=\mathrm{I}, 2, \ldots r .}
\end{array}
$$

For univariant systems $r=n+\mathrm{I}$. Accordingly, in this case, we may eliminate the $n$ quantities $d \mu_{\mathrm{r}}, d \mu_{2}, \ldots d \mu_{n}$ from the $n+\mathrm{I}$ equations (2). We obtain in this way Gibbs's first equation :

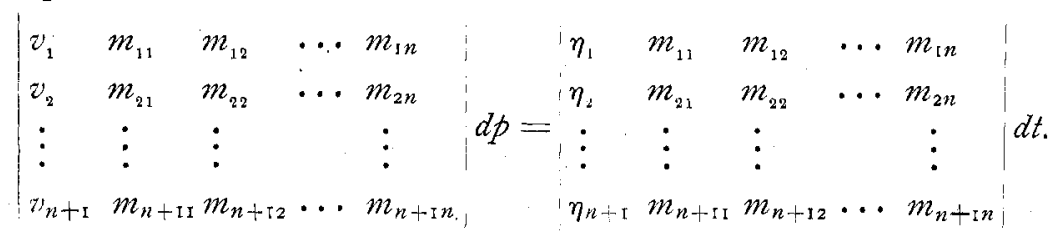

Writing the equations which express the fact that the total mass of a component in the system is equal to the sum of the masses of this component in the different phases, we have the $n$ equations :

$$
\mathrm{M}_{1} m_{1 j}+\mathrm{M}_{2} m_{2 j}+\cdots+\mathrm{M}_{n+1} m_{n+1 j}=\mathfrak{N}_{j}, \quad[j=\mathrm{1}, 2, \ldots n .
$$

These $n$ equations are not sufficient to determine the $n+\mathrm{I}$ quantities $M_{i}$. If we denote by $M_{i}$ and $M_{i}^{\prime}$ two sets of solutions of equations (4), it follows that

$$
\begin{array}{r}
\left(\mathrm{M}_{1}^{\prime}-\mathrm{M}_{1}\right) m_{1 j}+\left(\mathrm{M}_{2}^{\prime}-\mathrm{M}_{2}\right) m_{2 j}+\cdots+\left(\mathrm{M}_{n+1}^{\prime}-\mathrm{M}_{n+\mathrm{I}}\right) m_{n+1 j}=0, \\
{[j=\mathrm{I}, 2, \cdots n . \quad(5)}
\end{array}
$$

Moreover, if we denote the volume and the entropy corresponding to $\mathrm{M}^{\prime}{ }_{i}$ by $\mathrm{V}^{\prime}$ and $\mathrm{H}^{\prime}$ we have

$$
\begin{gathered}
\mathrm{M}_{1} v_{1}+\mathrm{M}_{2} v_{2}+\cdots+\mathrm{M}_{n+1} v_{n+1}=\mathrm{V} \\
\mathrm{M}_{2}^{\prime} v_{1}+\mathrm{M}_{2}^{\prime} v_{2}+\cdots+\mathrm{M}_{n+1}^{\prime} v_{n+1}=\mathrm{V}^{\prime},
\end{gathered}
$$

from which it follows immediately that 


$$
\begin{array}{r}
\left(\mathrm{M}_{1}^{\prime}-\mathrm{M}_{1}\right) v_{1}+\left(\mathrm{M}_{2}^{\prime}-\mathrm{M}_{2}\right) v_{2}+\cdots+\left(\mathrm{M}_{n+1}^{\prime}-\mathrm{M}_{n+1}\right) v_{n+1} \\
=\mathrm{V}^{\prime}-\mathrm{V} .
\end{array}
$$

Similarly it can be shown that

$$
\begin{array}{r}
\left(\mathrm{M}_{1}^{\prime}-\mathrm{M}_{1}\right) \eta_{1}+\left(\mathrm{M}_{2}^{\prime}-\mathrm{M}_{2}\right) \eta_{2}+\cdots+\left(\mathrm{M}_{n+\mathrm{I}}^{\prime}-\mathrm{M}_{n+\mathrm{I}}\right) \eta_{n+\mathrm{I}} \\
=\mathrm{H}^{\prime}-\mathrm{H} .
\end{array}
$$

Eliminating the $n+$ I quantities $\mathrm{M}^{\prime}{ }_{i}-\mathrm{M}_{i}$ from the $n+2$ equations $(5),(6),(7)$, we find that

$$
\left|\begin{array}{cccccc}
\mathrm{H}^{\prime}-\mathrm{H} \mathrm{V}^{\prime}-\mathrm{V} & \circ & \circ & \cdots & \circ \\
\eta_{1} & v_{1} & m_{11} & m_{12} & \cdots & m_{1 n} \\
\eta_{2} & v_{2} & m_{21} & m_{22} & \cdots & m_{2 n} \\
\vdots & \vdots & \vdots & \vdots & & \vdots \\
\eta_{n+1} & v_{n+\mathrm{I}} & m_{n+1 \mathrm{I}} & m_{n+12} & \cdots & m_{n+1}
\end{array}\right|=0,
$$

and this determinant when expanded gives

$$
\begin{aligned}
& \left(\mathrm{H}^{\prime}-\mathrm{H}\right)\left|\begin{array}{ccccc}
v_{1} & m_{11} & m_{12} & \cdots & m_{\mathrm{I} n} \\
v_{12} & m_{21} & m_{22} & \cdots & m_{2 n} \\
\vdots & \vdots & \vdots & & \vdots \\
v_{n+\mathrm{I}} & m_{n \rightarrow \mathrm{It}} & m_{n+\mathrm{I} 2} & \cdots & m_{n+\mathrm{I} n}
\end{array}\right| \\
& =\left(\mathrm{V}^{\prime}-\mathrm{V}\right)\left|\begin{array}{ccccc}
\eta_{1} & m_{11} & m_{12} & \cdots & m_{\mathrm{I} n} \\
\eta_{2} & m_{21} & m_{22} & \cdots & m_{2 n} \\
\vdots & \vdots & \vdots & & \vdots \\
\eta_{n+\mathrm{I}} & m_{n-\mathrm{II}} & m_{n-\mathrm{I} 2} & \cdots & m_{n+\mathrm{I} n}
\end{array}\right|
\end{aligned}
$$

The comparison of equations (3) and (8) shows that

$$
\frac{d p}{d t}=\frac{\mathrm{H}^{\prime}-\mathrm{H}}{\mathrm{V}^{\prime}-\mathrm{V}},
$$

and this equation takes the well-known form

$$
\frac{d p}{d t}=\frac{Q}{t\left(V^{\prime}-V\right)},
$$

if we denote by $Q$ the quantity of heat which the univariant system absorbs in expanding from the volume $\mathrm{V}$ to the volume $\mathrm{V}^{\prime}$ at the constant temperature $t$. 
Next, suppose that the system considered is bivariant. In that case $r=n$, and accordingly from the $n$ equations (2) we can eliminate $n$ - I of the quantities $d \mu_{j}$, say $d \mu_{2}, d \mu_{3}, \ldots d \mu_{n}$. The result of the elimination is the second of Gibbs's equations:

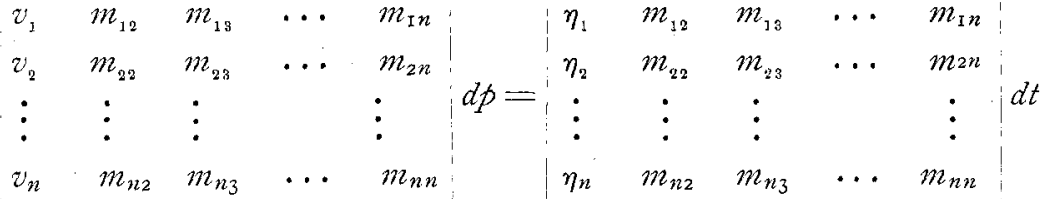

$$
\begin{aligned}
& \left|\begin{array}{cccc:}
m_{11} & m_{12} & \cdots & m_{\text {In }} \\
m_{21} & m_{22} & \cdots & m_{2 n} \\
\vdots & \vdots & & \vdots \\
m_{n 1} & m_{n 2} & \ldots & m_{n n}
\end{array}\right|
\end{aligned}
$$

We shall now show that, if

$$
\left|\begin{array}{cccc}
m_{11} & m_{12} & \cdots & m_{1 n} \\
m_{21} & m_{22} & \cdots & m_{2 n} \\
\vdots & \vdots & & \vdots \\
m_{n 1} & m_{n 2} & \cdots & m_{n n}
\end{array}\right|=0
$$

equation (II) can be put into the form of the Clapeyron-Clausius equation.

When equation (I2) is satisfied, the $n$ equations

$$
\mathrm{M}_{1} m_{1 j}+\mathrm{M}_{2} m_{2 j}+\cdots+\mathrm{M}_{n} m_{n j}=\mathfrak{N}_{j}[j=1,2, \ldots n .
$$

give infinite values for the quantities $M_{i}$, unless the following $n$ conditions are also verified :

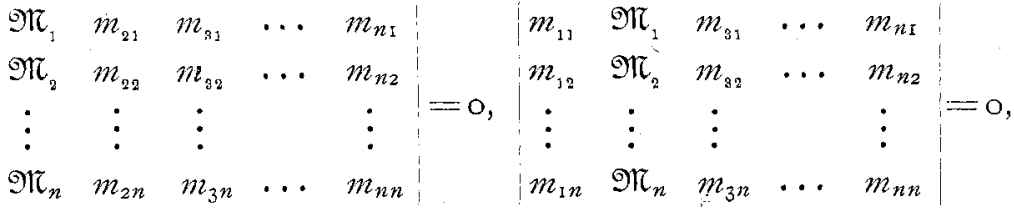

$$
\begin{aligned}
& \left|\begin{array}{ccccc}
m_{11} & m_{22} & m_{31} & \ldots & \mathfrak{K}_{1} \\
m_{12} & m_{22} & m_{32} & \ldots & \mathfrak{N}_{2} \\
\vdots & \vdots & \vdots & & \vdots \\
m_{1 n} & m_{2 n} & m_{n_{3}} & \ldots & \mathfrak{\mathscr { N }}_{n}
\end{array}\right|=0
\end{aligned}
$$


These $n$ homogeneous linear equations for the quantities $\mathfrak{M}_{j}$ are compatible. For if we form their discriminant it is at once apparent that the various terms in the resulting determinant are respectively the minors of the determinant of equation (I2). The discriminant is thus a power of the determinant in equation (I2), and it is accordingly equal to zero.

If we suppose that the masses $\mathscr{N}_{j}$ have values which satisfy equations (I4), the equations (I 3 ) are no longer sufficient to determine the masses $M_{i}$. We know, from the theory of linear equations, that we may replace one of the equations (I3), say the first, by the equation

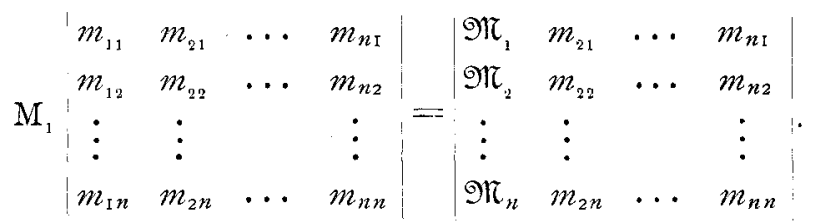

But as the determinants in this equation are equal to zero, $M_{r}$ may be chosen arbitrarily. The remaining $n-I$ equations of the group (I 3 ) will then determine the $n$ - I masses $M_{2}, M_{3}, \ldots$ $\mathrm{M}_{n}$. The first equation of the group (I3) is thus a consequence of the others.

Thus, when equations. (I2) and (I4) are verified, the bivariant system presents the following characteristics of a univariant system : the temperature and pressure are connected by an equation, equation (I2), and the masses of the phases may vary while the pressure and temperature remain constant. The bivariant system is then said to be in an indifferent state.

If $\mathrm{M}^{\prime}{ }_{i}, \mathrm{M}_{i}$ be two sets of solutions of equations ( $\mathrm{I} 3$ ), we have $\begin{aligned}\left(\mathrm{M}_{1}^{\prime}-\mathrm{M}_{1}\right) m_{1 j}+\left(\mathrm{M}_{2}^{\prime}-\mathrm{M}_{2}\right) m_{2 j}+\cdots+ & \left(\mathrm{M}_{n}^{\prime}-\mathrm{M}_{n}\right) m_{n j}=\mathrm{o}, \\ & {[j=2,3, \cdots n .}\end{aligned}$

and also the following equations analogous to equations (6) and (7),

$$
\begin{array}{r}
\left(\mathrm{M}_{1}^{\prime}-\mathrm{M}_{1}\right) v_{1}+\left(\mathrm{M}_{2}{ }^{\prime}-\mathrm{M}_{2}\right) v_{2}+\cdots+\left(\mathrm{M}_{n}{ }^{\prime}-\mathrm{M}_{n}\right) v_{n} \\
=\mathrm{V}^{\prime}-\mathrm{V} \\
\left(\mathrm{M}_{1}^{\prime}-\mathrm{M}_{1}\right) \eta_{1}+\left(\mathrm{M}_{2}{ }^{\prime}-\mathrm{M}_{2}\right) \eta_{2}+\cdots+\left(\mathrm{M}_{n}{ }^{\prime}-\mathrm{M}_{n}\right) \eta_{n} \\
=\mathrm{H}^{\prime}-\mathrm{H} .
\end{array}
$$


Eliminating the $n$ quantities of $\mathrm{M}_{i}^{\prime}-\mathrm{M}_{i}$ from the $n+\mathrm{I}$ equations ( I $_{5}$ ), (I6), and (I7), we get

$$
\begin{array}{cccccc}
\mathrm{H}^{\prime}-\mathrm{H} & \mathrm{V}^{\prime}-\mathrm{V} & \circ & 0 & \cdots & 0 \\
\eta_{1} & v_{2} & m_{12} & m_{13} & \cdots & m_{1 n} \\
\eta_{2} & v_{2} & m_{22} & m_{23} & \cdots & m_{2 n} \\
\vdots & \vdots & \vdots & \vdots & & \vdots \\
\eta_{n} & v_{n} & m_{n 2} & m_{n 3} & \cdots & m_{n n}
\end{array}
$$

which, expanded, becomes

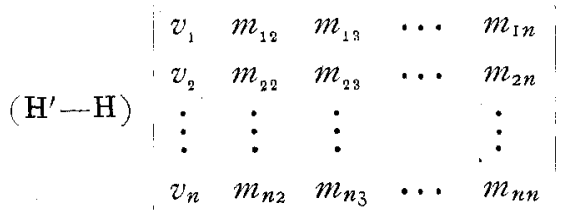

$$
\begin{aligned}
& =\left(\mathrm{V}^{\prime}-\mathrm{V}\right)\left|\begin{array}{ccccc}
\eta_{1} & m_{12} & m_{18} & \cdots & m_{1 n} \\
\eta_{1} & m_{22} & m_{23} & \cdots & m_{2 n} \\
\vdots & \vdots & \vdots & & \vdots \\
\eta_{n} & m_{n 2} & m_{n 3} & \cdots & m_{n n}
\end{array}\right|
\end{aligned}
$$

The comparison of equations (I8) and (II), in which the last term now disappears, shows that

$$
\frac{d p}{d t}=\frac{\mathrm{H}^{\prime}-\mathrm{H}}{\mathrm{V}^{\prime}-\overline{\mathrm{V}}},
$$

or

$$
\frac{d p}{d t}=\frac{\mathrm{Q}}{t\left(\mathrm{~V}^{\prime}-\mathrm{V}\right)},
$$

if we denote by $Q$ the quantity of heat which the bivariant system in the indifferent state absorbs in expanding from the volume $\mathrm{V}$ to the volume $\mathrm{V}^{\prime}$ at the constant temperature $t$. This equation completes the analogy which exists between the behavior of a univariant system and the behavior of a bivariant system in an indifferent state.

In this connection, it is interesting to note that the demonstration which Clapeyron gives of his formula is as general as it 
is simple. His reasoning applies to any system of constant mass whose state is determined by its pressure and volume. But one of the consequences of the phase rule is that, with the one exception of invariant systems, the state of every system of constant mass is determined by its pressure and volume. The formula which we have established is thus a special case of Clapeyron1's theorem, and as such cannot be called new; nevertheless it remained unnoticed until quite recently.

Bordeaux, January, Igoo. 